\title{
On cavitation in liquid helium in a flow due to a vibrating quartz fork
}

\author{
M. Blažková ${ }^{1}$, D. Schmoranzer ${ }^{2}$, and L. Skrbek ${ }^{1,2}$ \\ ${ }^{1}$ Joint Low Temperature Laboratory Institute of Physics ASCR, Na Slovance 2, 18221 Prague, Czech Republic \\ ${ }^{2}$ Faculty of Mathematics and Physics, Charles University, V Holešovičkách 2, 18000 Prague, Czech Republic \\ E-mail: skrbek@fzu.cz
}

Received November 27, 2007

\begin{abstract}
Cavitation in normal and superfluid liquid ${ }^{4} \mathrm{He}$ at saturated vapor pressure and slightly elevated pressures has been experimentally studied in a flow due to quartz forks vibrating at high amplitudes. Above the temperature- and pressure-dependent critical velocity, heterogeneous cavitation is observed both visually and electrically, as a breakdown of the resonance response of the fork. We compare our results with available experimental and discuss them using existing theoretical models. In particular, we show that thermal effects leading to local overheating of the vicinity of the fork have to be taken into account, especially in normal liquid ${ }^{4} \mathrm{He}$.
\end{abstract}

PACS: $47.55 . d p$ Drop and bubble formation.

Keywords: cavitation, He I, He II, quartz tuning fork.

\section{Introduction}

Cavitation generally means nucleation of bubbles inside a liquid. Due to the fact that the liquid-gas phase transition is of the first order, a liquid can, for a finite time, stay in a metastable state outside the stability region in the equilibrium phase diagram - it is known that liquids can often be stretched to large negative pressures (in the case of pure water down to about -1400 bar [1]). For a more stable gas phase to appear inside the metastable liquid, an interface of a finite surface energy has to be created and, as a consequence, there is an energy barrier that has to be overcame in order for cavitation to occur.

In common liquids nucleation of the gaseous phase is usually affected by the presence of impurities, dissolved gases, defects, walls or radiation - all these factors make nucleation easier and in this case cavitation is called heterogeneous. Cavitation is called homogeneous, if it appears as an intrinsic property of a pure system - in this case it may occur very far from the thermodynamic equilibrium.

Liquid helium can be prepared extremely clean, wets almost ideally any solid surface and is therefore considered as a useful and interesting model system for studying cavitation (including quantum cavitation). Nucleation of bubbles inside a liquid is usually called boiling when it re- sults from a temperature change; the term cavitation is used when bubbles occur due to a pressure change. These two processes, however, belong to the same physics and, as we shall see later, in most cases ought to be considered simultaneously in liquid helium. Despite numerous studies on cavitation that have been performed over the last fifty years (see Ref. 2 for a comprehensive review of the early experiments on nucleation of bubbles in liquid helium, and Ref. 3 for the more recent results; for a broader picture see a nice review in Ref. 4 by Balibar), cavitation processes in liquid ${ }^{4} \mathrm{He}$ — both in $\mathrm{He}$ I and $\mathrm{He}$ II — are only partly understood. It is therefore of considerable interest to revisit this field using a new tool - a commercially available quartz tuning fork, mass-produced as a frequency standard for digital watches.

The vibrating quartz tuning fork has proved as a robust, cheap, widely available and easy to use multipurpose tool for low-temperature physics and cryogenic fluid dynamics [5], in particular for generating and probing cryogenic oscillatory boundary layer flows of gaseous and liquid helium. The basic physical properties of the flow due to such a vibrating fork driven electrostatically at low amplitudes (in the linear flow regime) in gaseous and in liquid helium (including superfluid $\mathrm{He}$ II and superfluid ${ }^{3} \mathrm{He}-B$ ) have been reported in Ref. 6 , where the 
calibration procedure and the potential of the fork used as thermometer or pressure gauge has been considered in detail. Moreover, if driven in the nonlinear flow regime, it serves as an excellent tool to study the crossover from the laminar drag regime (characterized by a linear drive versus velocity dependence) to the turbulent drag regime (characterized by a quadratic drive versus velocity dependence) in both classical viscous fluids [7] and quantum fluids [8].

The peak velocity of a tuning fork vibrating in helium fluids can be easily varied and detected over seven orders of magnitude, up to very high values of order $\mathrm{m} / \mathrm{s}$, limited by its mechanical and/or electrical strength. Using an identical experimental setup as described in Refs. 6, 7 this enables us to observe experimentally additional effects (see a preliminary report [9]) in liquid He I and He II (absent in gaseous $\mathrm{He}$ ), which we ascribe to cavitation taking place in the vicinity of the vibrating fork. This report accounts for our experimental results and current understanding of the underlying physics.

The paper is organized as follows: In Sec. 2 we shortly describe our tools - quartz tuning forks and the experimental apparatus. In Sec. 3 our visual and electrical observations of cavitation are presented. In Sec. 4 we compare our data with previous studies on cavitation and discuss them within available physically motivated models. We conclude in Sec. 5.

\section{Forks and experimental apparatus}

Commercially produced quartz tuning forks vibrating at the frequency of $2^{15} \mathrm{~Hz}(=32768 \mathrm{~Hz})$ at room temperature, specified as type DT26 (A1, A2) and DT38 (B1, B6) (Fronter Electronics, China, www.chinafronter.com) have been used in this work. They are supplied in cylindrical vacuum-tight metal cans that have to be entirely or partly removed. When cooled to liquid helium temperature, their frequency linewidth measured in vacuum is typically only about $0.05 \mathrm{~Hz}$, resulting in the quality factor of order $10^{5}$. For visual observation of cavitation, we used bare forks (i.e., with their cans entirely removed) in an open helium bath in a standard glass cryostat placed in- side an additional glass liquid nitrogen cryostat. For electrical measurements, we have additionally used forks with only the top of their cans ground off, positioned vertically pointing upwards, so that the buoyancy force enables an easy escape of gas bubbles from inside the can. Although the open bath was used for electrical measurements as well, most of our electrical measurements were performed inside a small cylindrical brass pressure cell. The forks were soldered via their electrical leads to the ceramic feedthrough in the bottom of the cell, which is then screwed to the body of the cell and sealed by an In o-ring. The cell was tested to withstand pressures up to 30 bar at low temperatures and can be pressurized via a thin capillary and a copper sinter placed at its inlet in situ, using a charcoal cryogenic pressurizer.

The electrical circuit used for the detection of cavitation by the vibrating quartz fork is schematically shown in Fig. 1. It allows monitoring the response of the fork over seven orders of magnitude of the drive. For cavitation to occur, a very high drive amplitude of order 100 Vrms is needed (in He II), which is achievable by using a step-up transformer (30 times) on the output of the Agilent 33250A function generator. Such a high drive is close to the critical level at which the fork breaks mechanically and/or electrically, and, indeed, a number of forks were destroyed during this work. To detect the in-phase and out-of-phase signal simultaneously, we use the dualphase SR830 lock-in amplifier. The applied driving voltage and the measured electrical signal are converted to the driving force and the fork velocity using the calibration procedure described in detail in Ref. 6. To obtain temperatures $\simeq 1.3 \mathrm{~K}<T<4.2 \mathrm{~K}$, we use a powerful pumping unit based on CIT Alcatel RSV 350 Roots pump. When needed, the temperature of the helium bath is stabilized using the Conductus LTC 21 temperature controller. The absolute pressure in the pressure cell is accurately measured by the MKS Baratron 690A pressure meter; care is taken to add the hydrostatic pressure head due to the liquid helium column, the height of which above the fork is assumed to be the same in the cell filling capillary as monitored in the helium bath of the glass cryostat.

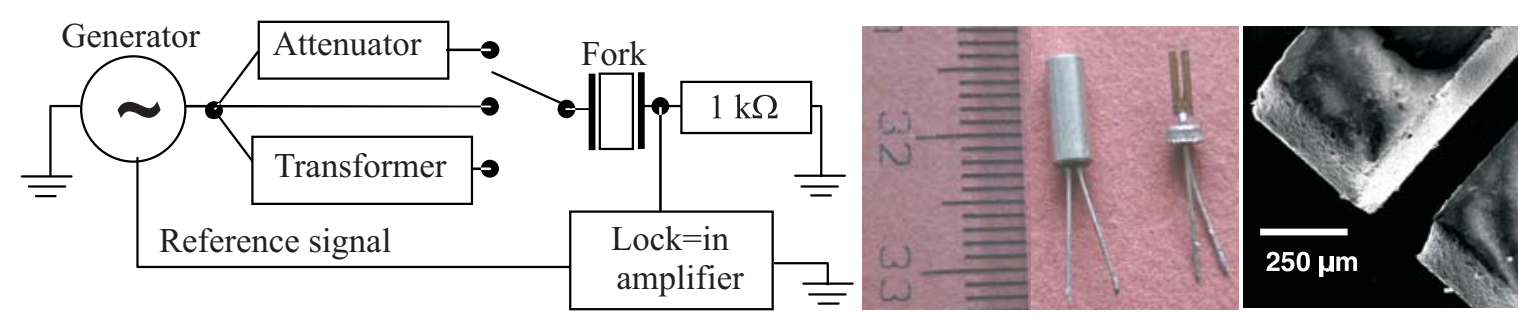

Fig. 1. The electrical circuit diagram used to detect cavitation with the tuning fork. With the help of the step-up transformer, an ac driving signal of up to $130 \mathrm{~V}_{\text {rms }}$ can be applied (left). The photographs of the bare and encapsulated forks (middle) and the detail of the sharp corners of their prongs (right). 


\section{Electrical detection and visual observation of cavitation}

\subsection{Experimental protocol and electrical data on cavitation}

Our detection protocol is based on sweeping the fixed driving voltage applied to the quartz tuning fork, of the form $U=U_{0} \cos \omega t$, across the resonance peak. At low driving voltages (after adjusting the phase) the response signal of the fork consists of the absorption and dispersion curves of Lorentzian form [6]. On increasing the driving voltage, however, the response ceases to be Lorentzian, the absorption resonant curve widens and the maximum response shifts towards lower frequency. In gaseous and liquid ${ }^{4} \mathrm{He}$, where the viscous penetration depth $\delta=\sqrt{2 v / \omega}$ is much smaller than any linear dimension of the fork we have shown experimentally that, over two orders of magnitude of kinematic viscosity $v$ and over a decade in fluid density $\rho$ this transition can be characterized by a critical velocity which scales as $U_{\text {cr }} \propto \sqrt{v \omega}$, where $\omega$ is the angular frequency of oscillations. These features are universal in that they occur irrespectively of whether the fork is driven in He I, He II or in gaseous helium [7].

In liquid $\mathrm{He}$ I and $\mathrm{He}$ II additional pronounced features occur along their saturated vapour pressure curve (SVP) or at slightly elevated pressures, as illustrated in Fig. 2. For a high enough drive amplitude the observed signal breaks down when the frequency is swept up, first just before reaching the expected maximum in the absorption signal. This event serves us as a definition of the critical cavitation velocity $v_{\text {cav }}$ - the velocity at which the breakdown of the signal happens. Unless otherwise stated, we use the rms velocity throughout this paper. When the frequency sweep of the drive is continued slowly (a typical time scale of a sweep is a minute), in most cases the signal recovers at approximately the same frequency difference past the expected maximum (see Fig. 2, middle). When sweeping across the resonance with an even higher drive level, the destroyed part of the Lorentzian-like response broadens and the recovery point is not in most cases symmetric with respect to the ex-
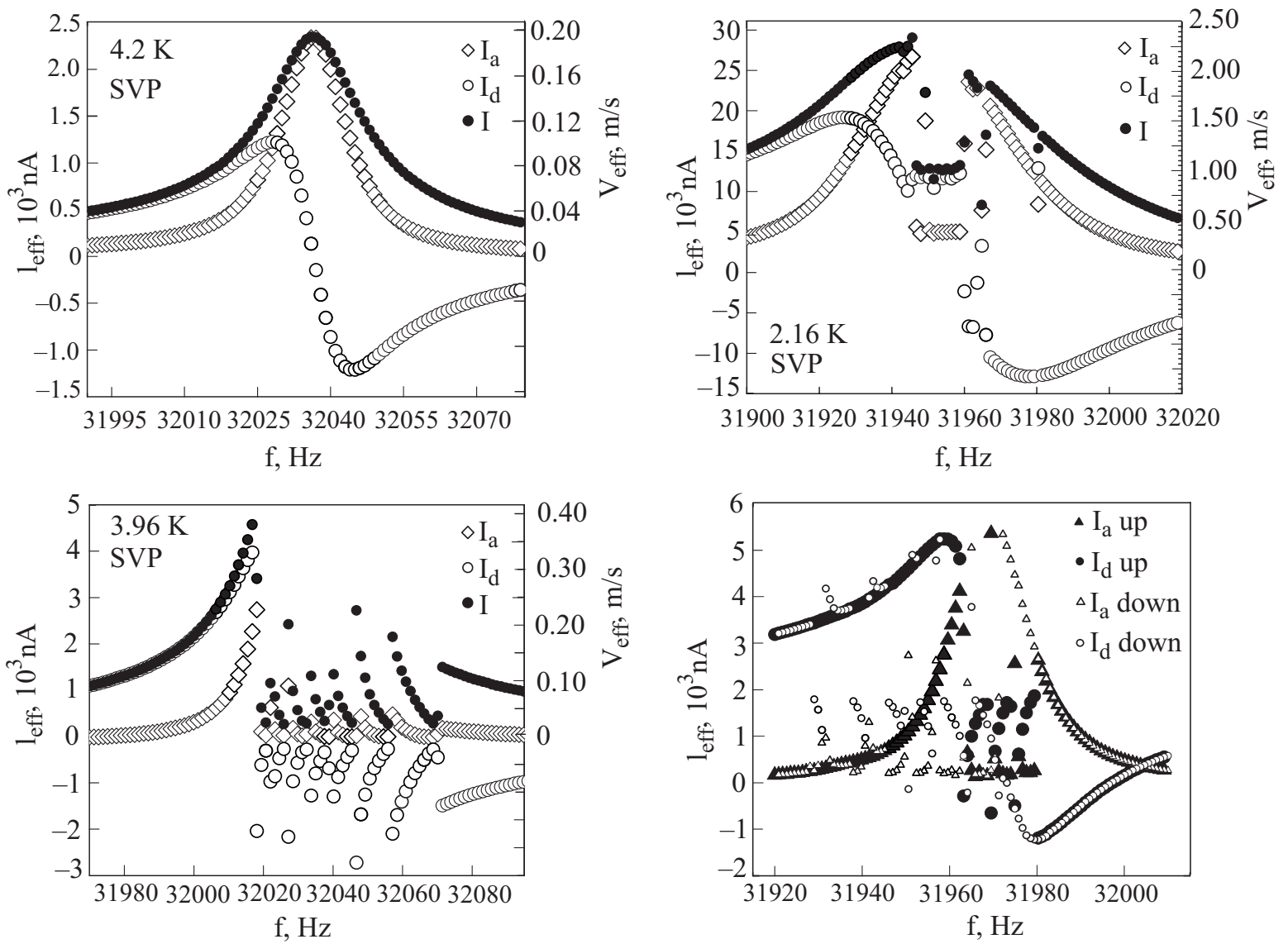

Fig. 2. The amplitude $(\bullet)$, in-phase $(\diamond)$ and out-of-phase $(\bigcirc)$ signal obtained when sweeping the driving voltage across its resonant response. Up to a critical drive level, the observed curves are of nearly Lorentzian shape (top left). For higher drive amplitudes, on approaching the expected maximum the observed signal drops abruptly and recovers when the slow sweep passes the maximum response region (top right). This form of the signal is typical in He II. The signal in He I is typically of a more complex form and includes a series of abrupt peaks followed by nearly exponential decays, before re-joining the original Lorentzian curve (bottom left). The difference in the absorption and dispersion signals for up and down frequency sweeps in He I (bottom right). 
pected maximum response any more. These described forms of the signal can be observed both in He I and He II.

The signal in He I can be of a more complex form and might include a series of abrupt peaks followed by nearly exponential decays, before re-joining the original Lorentzian curve (see Fig. 2, bottom left). When repeating the sweeps, this peculiar character of the signal persists but generally, the response is not exactly reproducible. This character of the signal has been observed both in up and down frequency sweeps (see Fig. 2, bottom right). The mirror image character of the signal observed in up and down frequency sweeps suggests that the approximately exponential decaying parts following the peaks are in fact temporal decays of the observed response of the fork.

Figure 3 shows the temperature dependence of the cavitation velocity (defined as explained above), as observed in different runs for various geometries (bare fork in the open helium bath, partly encapsulated fork inside the pressure cell) of the flow due to the vibrating fork. On decreasing the temperature below $4.2 \mathrm{~K}$ towards the $\lambda$-point $v_{\text {cav }}$ increases slowly, especially if one considers the data series obtained inside the pressure cell for a tuning fork inside its partly opened original can. Measurements performed with the bare fork inside the pressure cell and using the bare fork directly in the open helium bath of the glass cryostat are in qualitative agreement, but display a somewhat larger scatter of the data.

The most striking feature of these data is a very steep increase in $v_{\text {cav }}$ right below the $\lambda$-point - here $v_{\text {cav }}$ rises by factor of $3-5$. We have measured this pronounced feature with several forks; it displays a reasonable degree of reproducibility for the data series from different runs as well as for the data obtained with different forks. We be-

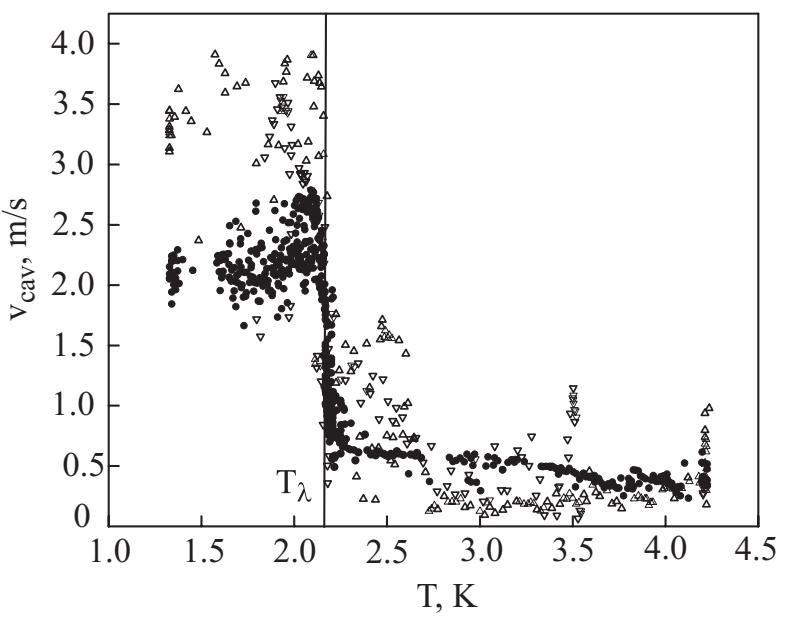

Fig. 3. The observed critical cavitation velocity plotted versus the temperature at the saturated vapour pressure using a partly encapsulated fork (lidless cup geometry) inside the pressure cell (filled circles), a bare fork inside the pressure cell (up triangles) and a bare fork in the open helium bath of the glass cryostat (down triangles). lieve that this abrupt increase in $v_{\text {cav }}$ below the $\lambda$-point is therefore firmly established.

Further reduction of temperature down to about $1.3 \mathrm{~K}$ seems to have much less effect on $v_{\text {cav }}$; within the rather large scatter it stays roughly constant, but statistically a maximum can be allocated to about $1.9 \mathrm{~K}$.

Having available the pressure cell $[6,8]$, we have performed the measurements showing the dependence of $v_{\text {cav }}$ versus an externally applied overpressure at 4.2, 2.9 and $1.3 \mathrm{~K}$ (see Figs. 4, 8 and 9). As expected, it is evident that at all temperatures the observed $v_{\text {cav }}$ increases with the applied overpressure.

\subsection{Visual observation of cavitation}

It is important to confirm independently that the described effects are indeed caused by cavitation. The unequivocal proof is the direct visual observation of a bubble between the prongs of the fork in He II, where there are no bubbles in the bulk thanks to the extremely large thermal conductivity. Figure 5 shows a digital photograph of such a bubble. In the glass cryostat, we have clearly observed the bubble in the space between the prongs of the fork, attached to the surface of one of them or, in some cases, the bubble between the prongs was attached to both of them. During the frequency sweeps such as shown in the top right panel of Fig. 2, the bubble appears when $v_{\text {cav }}$ is reached, exists as long as the otherwise Loretzian response is broken and disappears when the Lorentzian-like response recovers. We have recorded several digital video sequences of this process, clearly showing the wiggling bubble attached to the fork, confirming the described scenario. The bubbles were observed clearly and easily in the open bath cryostat only within the temperature range about $2.07 \mathrm{~K}<T<2.14 \mathrm{~K}$, although the electrical signal outside this range does not appear to differ qualitatively.

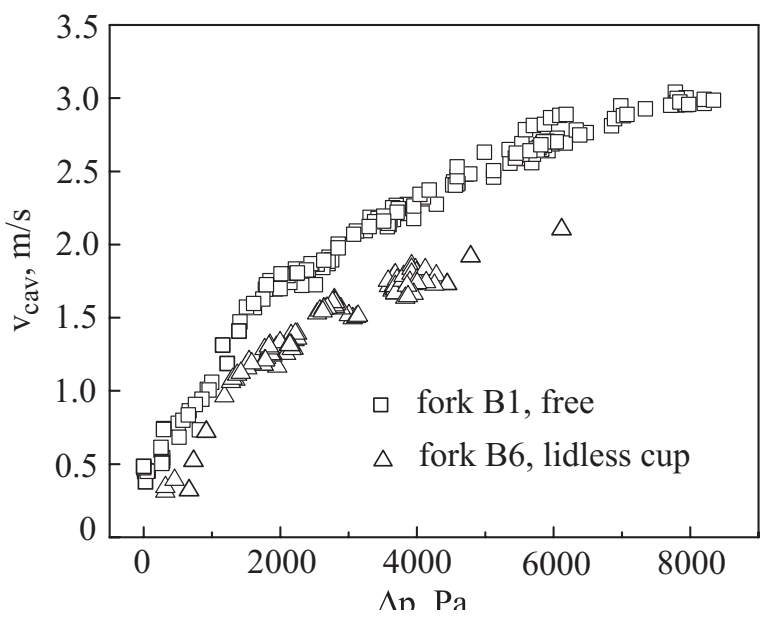

Fig. 4. The observed critical cavitation velocity plotted versus the applied overpressure at $4.2 \mathrm{~K}$ for two forks of the same type in different geometries: fork B1 is bare, fork B6 in a lidless cup, both inside the pressure cell. 


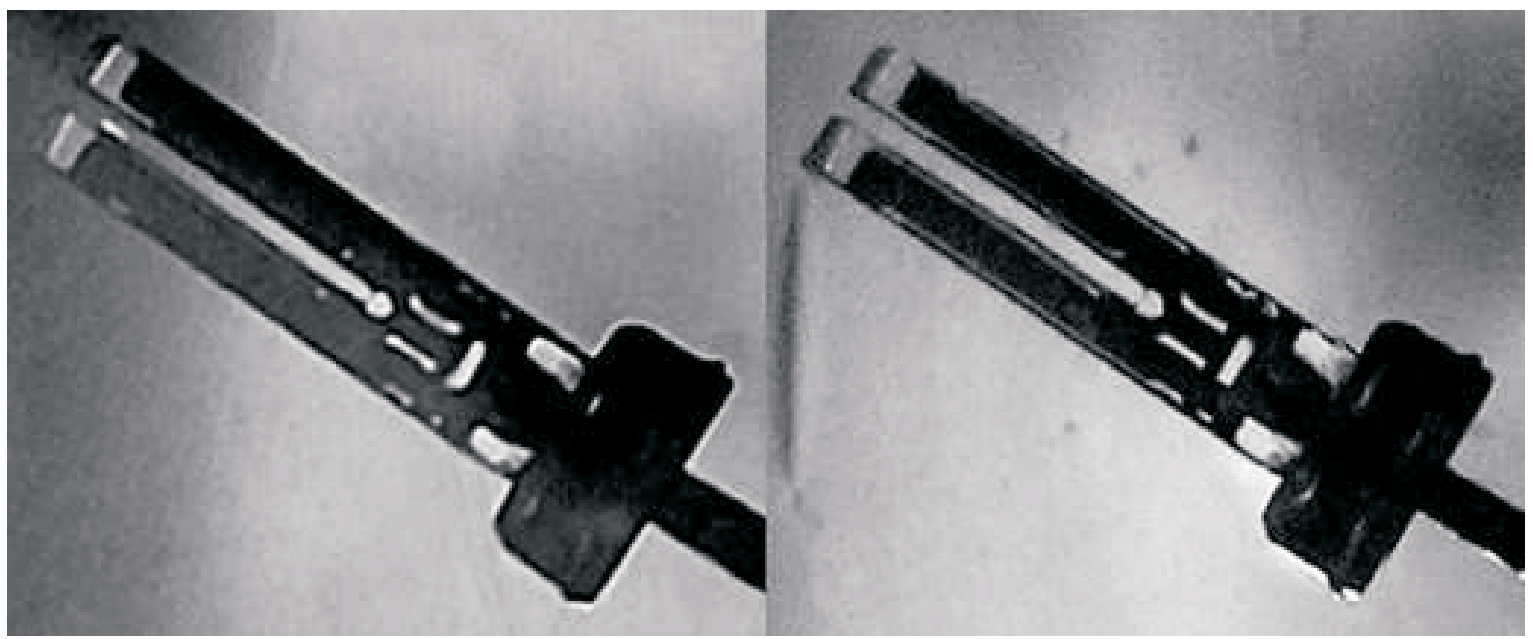

Fig. 5: Left: A digital photograph of the fork vibrating at a high amplitude in the He II bath kept at $T=2.14 \mathrm{~K}$, showing the elliptically shaped bubble between its prongs, attached to the lower one. Right: The photograph of the same fork without a bubble, taken a few moments later for comparison.

In He I kept at the saturated vapour pressure any visual evidence of cavitation is as yet inconclusive, due to the small bubbles always present in the boiling helium bath in the glass cryostat. We have not been able to distinguish convincingly between these bubbles and those that would originate in the vicinity of the vibrating fork due to the otherwise clearly electrically indicated cavitation process.

\section{Discussion}

First of all, we emphasize that the «breakdown» effects described above never occurred when driving the fork in vacuum or in gaseous helium at any temperature and applied pressure. Based on this fact and the above mentioned visual observation in He II, it is natural to assume that these «breakdown» effects arise as a consequence of cavitation/boiling processes, both in He I and He II.

We shall start our discussion with He II, where cavitation is confirmed by visual observation. We shall continue with our temporary considerations relevant to the observed effects in He I. We shall see that, thanks to the extremely large thermal conductivity, any possible local overheating of He II in the vicinity of the vibrating fork could be neglected and the flow could be treated as isothermal, at least approximately. In the poorly conducting He I we shall have to take thermal effects into consideration. Moreover, we shall see that thermal effects might give useful hints for various remaining cavitation puzzles hidden in the experimental data of other investigators. While discussing our own data, it seems useful to revisit some of the plentiful existing data and compare them to our own.

\subsection{Previous studies on cavitation in liquid helium}

The first quantitative measurement of the tensile strength of liquid helium belongs to Beams [10], who noted that the tensile strength he measured $(0.14$ bar at $1.8-1.9 \mathrm{~K})$ was far lower than the expected value.

Several experiments using planar [11] or tubular $[12,13]$ piezoelectric transducers operated at frequencies similar to ours led to the observation of cavitation in liquid helium. Finch, Kagiwada, Barmatz and Rudnick [11] used two identical disks $4.5 \mathrm{~cm}$ apart, made of Clevite PZT-4 ceramics $1 / 2$ inch in diameter and thickness, with electrodes on their flat surfaces operated at $91.15 \mathrm{kHz}$, one as a transducer, the other as a receiver, to generate and detect cavitation in bulk helium. Cavitation was detected via the acoustic emission associated with the collapse of bubbles, and also visually, albeit at much higher drive amplitudes. The deduced acoustic cavitation thresholds were generally low, comparable to the static pressure head in the helium bath. The procedure of establishing the acoustic threshold was to increase the drive to such a level when the cavitation sound was heard continuously and then reduce it to the point when a burst of sound could not be heard more often than once every five seconds. This so-called acoustic threshold was found roughly independent of temperature in He II, but when approaching the $\lambda$-point from below, within about $0.01 \mathrm{~K}$, a sharp peak (up to about 5 times its low temperature value) was detected.

In subsequent experiments, Finch and Wang [14] clarified two types of cavitation in liquid helium occurring at frequencies (40-100) kHz: (i) an acoustic type of cavitation detected by cavitation noise caused by nonlinear interaction between the sound field and the microscopic bubbles acting as active scattering centers and (ii) a visible type of cavitation that can be seen with the naked eye and produces its own rather harsher kind of noise. Below 
$2.13 \mathrm{~K}$ the acoustic cavitation threshold was about ten times lower than the visible one, between $2.13 \mathrm{~K}$ and the $\lambda$-point the visible threshold decreased and in $\mathrm{He} \mathrm{I}$ the two coincided, being comparable to the acoustic one in He II. It is important to notice that only the visible threshold was affected by variations in the externally applied static pressure [15].

Our experiment with quartz fork whose prongs oscillate as cantilevers in antiphase against each other at the frequency of about $32 \mathrm{kHz}$ is geometrically similar to the experiments of Finch and coworkers [11], in that both experiments feature oscillations of solid surfaces perpendicularly to their plane. However, there is an important difference. The frequency of about $90 \mathrm{kHz}$ [11] corresponds to a wavelength of about $3 \mathrm{~mm}$, thus the space between the plane ceramics ( $4.5 \mathrm{~cm}$ apart) could act as an acoustic resonator, where both positive and negative pressure amplitudes might be enhanced strongly thanks to the constructive interference of the emitted acoustic waves. A similar improved technique, using a cylindrical acoustic standing wave of frequency $50.58 \mathrm{kHz}$ at $T=2.09 \mathrm{~K}$ (note that our visual observation was possible only in a narrow temperature window containing this temperature) was implemented by Marston [13]. Bubbles appeared to originate in pressure antinodes, expanded to a large diameter of $0.5-1 \mathrm{~mm}$ and eventually fragmented into smaller bubbles.

In the case of the fork oscillating at about $32 \mathrm{kHz}$, the span between the prongs is about 50 times shorter than the wavelength, so it cannot act as an acoustic resonator. In fact, we can rule out any other possible resonant enhancement of the emitted acoustic wave as a source of cavitation for the following reason: we have performed experiments with an almost fully encapsulated fork as well as with an entirely bare fork in the open He II bath, with results only slightly differing from each other (see Fig. 3). This allows excluding acoustic interference from our considerations.

Other important factors for consideration are thought to be the volume and the purity of the liquid where cavitation might occur [2]. In large volumes, the observed cavitation is usually heterogeneous in nature, affected by the presence of walls, vortices, impurities, radiation, etc. Indeed, the early experiments as well as our own were performed in technical helium with no extra care taken to purify it, so it most likely contained frozen air particles, positive and/or negative ions generated by cosmic rays, normal and in the case of He II also quantized vortices. For example, in the experiment of Finch and coworkers [11] the volume where cavitation occurred was about $23 \mathrm{~cm}^{3}$. In modern experiments with hemispherical capacitors $[2,4]$ this volume is much smaller, about $(\lambda / 2)^{3} \simeq$ $\simeq 2 \cdot 10^{-12} \mathrm{~m}^{3}$ (note in passing that this volume is only an order of magnitude or so smaller than the volume between

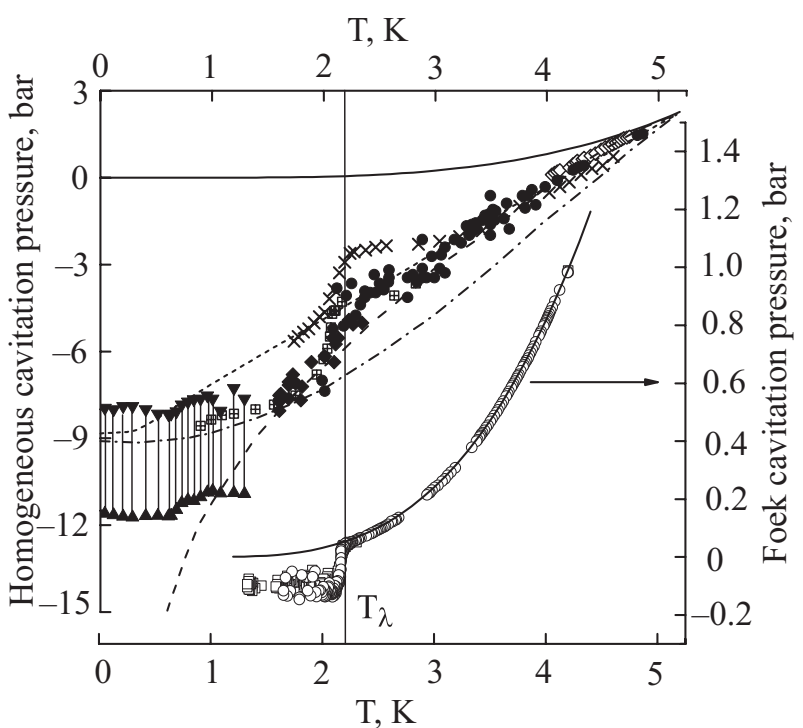

Fig. 6. The pressure-temperature phase diagram containing theoretical and experimental results on cavitation in liquid ${ }^{4} \mathrm{He}$. The two thick solid lines denote the equilibrium saturated vapour line with regard to the left and right vertical axes. The right axis is relevant to our own data (open circles and squares, bottom; see the text for our experimental method), the left axis pertains to the data of previous investigators: crosses - Ref. 16, squares with the plus symbol - Ref. 17, filled diamonds and circles - Ref. 18, empty diamonds - Ref. 19, filled triangles - Ref. 20. The experimental data of Ref. 20 clearly show the deviation from the prediction of the standard nucleation theory, denoted by the dashed line, which crosses even the spinodal limit (dash-dot line) and diverges at low temperatures, indicating the quantum nucleation regime. The dotted curve marks the position of the nucleation line calculated based on the density functional theory, see Refs. 21, 22.

the fork's prongs) and it has became a common notion that one deals with purely homogeneous nucleation here - the probability of heterogeneous nucleation far away from walls and due to the unlikely presence of nucleation sites in such a small volume is low. The main results of these experiments are shown in Fig. 6 and we shall discuss some of their aspects later. Here we only mention that they are in good qualitative agreement with the theoretical description of nucleation theories such as the so called thin wall approximation theory (where applicable) and the density functional theory (for further details, see Ref. 4 and references therein). It seems fair to say that homogeneous cavitation in He I and He II, including quantum cavitation at temperatures below $\sim 1 \mathrm{~K}$, is understood over the entire temperature range qualitatively. Quantitative agreement is quite good, except in the vicinity of the superfluid transition (see Fig. 6), where, as we shall discuss later, thermal effects ought to be taken into account. 


\subsection{Importance of thermal effects}

The thermal effects, namely heating the surrounding liquid due to the viscous damping of the fork vibrating at a high amplitude, may become highly important for cavitation experiments under certain experimental conditions. Figure 7 schematically shows why it ought to be so. The liquid next to the fork at rest placed at the depth $h$ below the liquid surface corresponds to the point $\left(\rho_{0}, T_{0}\right)$ of the equilibrium $p-T$ phase diagram; the liquid adjacent to the surface corresponds to the $\left(p_{S V P}, T_{0}\right)$ point. The pressure $p_{0}=p_{S V P}+\rho g h$. The externally applied overpressure would shift this value by $\Delta p_{\text {ext }}$ up, but the underlying physics does not change qualitatively and we shall therefore omit it in further discussion. The pressure $p_{S V P}$ is the equilibrium pressure at the saturated vapor curve (upper solid line marked SVP in Fig. 7) at $T=T_{0}$ - the temperature at which the helium bath is kept, measured some distance away from the fork. In order for (intrinsic) cavitation to occur in the vicinity of the fork, the lower thick solid line - the nucleation line - must be reached. The nucleation line can be reached, e.g., along the vertical arrow, by applying a locally lower or (below about $4 \mathrm{~K}$ ) even negative pressure. We shall call this process pure cavitation. It is clear, however, that the nucleation line could be reached also along the horizontal arrow, by locally overheating the vicinity of the fork, and we shall call this process pure boiling.

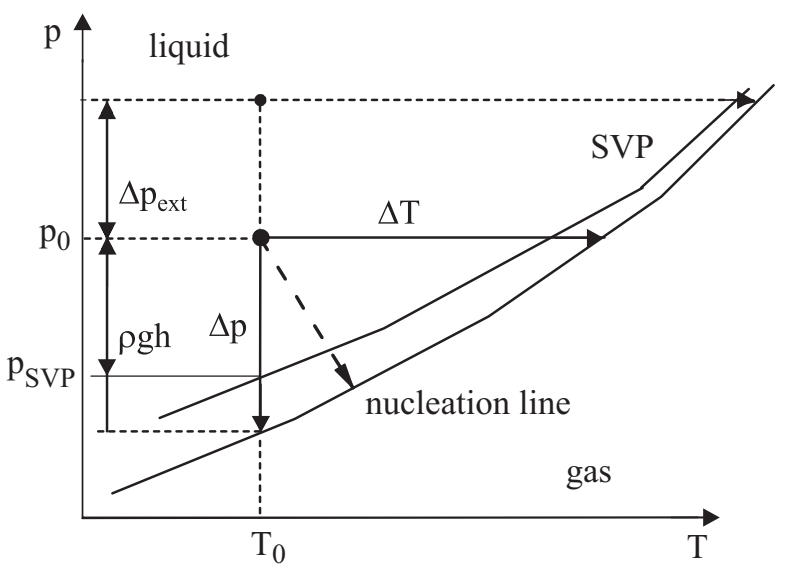

Fig. 7: The schematics of the underlying physical processes that could be relevant to the observed cavitation phenomena. The upper thick solid line represents the saturated vapor pressure (SVP) curve, dividing the area of the equilibrium $p-T$ phase diagram into gaseous and liquid phase regions. The oscillating fork is placed at depth $h$ below the liquid surface, resulting in $\Delta p=\rho g h$ above SVP, unless an additional external overpressure $\Delta p_{\text {ext }}$ is applied. When the fork oscillates, the nucleation line could be, in principle, reached from $\left(p_{0}, T_{0}\right)$ : (i) isothermally, by reducing the pressure as a consequence of the Bernoulli equation - the vertical arrow; (ii) by pure heating - the horizontal arrow; (iii) by a combination of both these processes as illustrated by the dashed arrow.
In practice, the nucleation line will most likely be reached by a combination of both of these processes, which is shown schematically by the dashed tilted arrow. The angle by which the arrow is tilted depends on the actual experimental conditions. It is clear, however, that the tilt will be much larger in He I, as the extremely high thermal conductivity of He II prevents any significant local overheating.

Let us now estimate the possible local overheating in our experiment with a partly encapsulated fork - with only the top of the encapsulating case (about $2 \mathrm{~mm}$ in diameter) ground off. In He II, cavitation occurs at a velocity $v_{\text {cav }} \cong 2 \mathrm{~m} / \mathrm{s}$. Based on our earlier measurements of the transition to turbulence in a flow due to a vibrating fork [7], we know that in steady state this velocity of the fork requires a driving force of about $F \simeq 5 \cdot 10^{-4} \mathrm{~N}$. Thus the heat influx to the surrounding liquid yields $\dot{Q} \approx F v_{\text {cav }} \approx 10^{-3}$ W. Assuming that this heat influx is carried away to the bulk by the outflowing normal fluid in a counterflow occurring inside the encapsulating case, we arrive at a heat flux per unit area of about $\dot{q}=\dot{Q} / A \approx 0.033 \mathrm{~W} / \mathrm{cm}^{2}$. This heat converts the incoming superfluid into the outflowing normal fluid, therefore $\dot{q}=V_{n} S T \rho$. At $T \approx 1.3 \mathrm{~K}(S \approx 0.085 \mathrm{~J} / \mathrm{gK})$, this leads to $V_{n} \approx 2 \mathrm{~cm} / \mathrm{s}$. At this temperature $\rho_{n} / \rho_{s}<<1$ and therefore this is also an estimate of the counterflow velocity (of the order of the critical velocity for the onset of counterflow turbulence) at the same time. At a high fork velocity (about $2 \mathrm{~m} / \mathrm{s}$ ), far beyond the onset of the turbulent drag regime [7], there is a dense tangle of quantized vortex lines in the boundary layer and the extra quantized vortices due to thermal counterflow would hardly matter. Any temperature gradients associated with this counterflow carrying the dissipated heat away from the fork are therefore probably very small and can be neglected. We are therefore very likely dealing with pure cavitation. We shall see later that close below the $\lambda$-point and in He I the situation is quite different.

\subsection{Discussion of our He II data}

Since we argued that the thermal effects can be neglected in He II, we are left to assume that, in order for cavitation to occur in our experiments, negative pressures must be reached at least locally. The first and perhaps naive idea which comes to mind is the application of the Bernoulli equation, according to which pressure is reduced in regions of high flow velocities. The highest flow velocities would be found in the vicinity of the sharp corners on the prongs of the tuning fork, where the flow is enhanced strongly. We are, of course, aware that the use of the Bernoulli equation for the description of a non-stationary and moreover turbulent flow is far from rigorous, but at least a simple test of this approach can be performed by analyzing the data we obtained in our overpressure experiments shown in Fig. 8. 


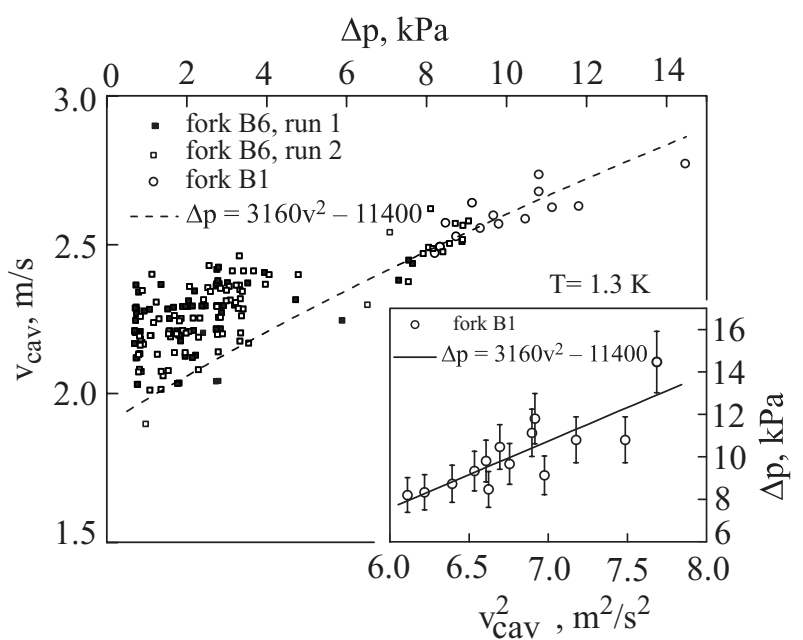

Fig. 8. The observed critical cavitation velocity plotted versus the applied overpressure in the cell at $1.3 \mathrm{~K}$. The dashed line is the best fit of the B1 fork data to the Bernoulli equation with parameters as given in the figure. The inset shows the dependence of the overpressure on the fork rms velocity squared which can be seen to be approximately linear, in accord with the Bernoulli equation.

Despite the considerable scatter, we see that the data qualitatively obey the Bernoulli equation $\Delta p=c v_{l}^{2} / 2$, where $v_{l}$ is the maximum velocity of the flow in the vicinity of the fork. This maximum flow velocity differs from the observed velocity of the prongs of the fork, $v_{\text {cav }}$, for two reasons. First, the flow velocity is enhanced hydrodynamically. Second, the relevant quantity for the occurrence of cavitation is not the rms velocity, but rather the peak velocity. In order to achieve quantitative agreement of our data with the prediction based on the Bernoulli equation, using the known value of He II density of $145 \mathrm{~kg} / \mathrm{m}^{3}$, a flow enhancement factor of approximately $k_{e}=4.7$ is needed. Is this flow enhancement feasible in He II?

The strongest enhancement can be expected in the close vicinity of the sharp corners of the fork's prongs, where He II passes right angles. In this case, the enhancement factor can be estimated [23] as $k_{e}=\sqrt[3]{L / r}$, where $L$ is the characteristic size of the prong in the direction of the flow and $r$ is the radius of curvature of the corner. Taking $L \approx 0.2 \mathrm{~mm}$, we arrive at $r \approx 2 \mu \mathrm{m}$.

In the case of an oscillatory classical viscous flow, even if the right-angled corners on the prongs were infinitely sharp, the effective radius of curvature would be of order of the viscous penetration depth $\delta=\sqrt{2 v / \omega}$. Despite the fact that we are dealing with He II, a similar quasiclassical approach can still probably be used, because cavitation occurs at velocities far beyond the transition to turbulence and it is known [24] that in this case He II can be described as a quasiclassical single-component fluid with an effective kinematic viscosity comparable to the kinematic viscosity of He I. Taking this into account, we find that the radius of curvature of $2 \mu \mathrm{m}$ agrees with the penetration depth within a factor of 2 . We therefore believe that the calculated enhancement factor can indeed be reached in our flow and our assumption that cavitation occurs in the vicinity of the sharp corners of the prongs seems plausible.

These considerations lead to a cavitation pressure of about $-11 \mathrm{kPa}$, which certainly corresponds to negative absolute pressure at $T=1.3 \mathrm{~K}$. Note that this value agrees with the early measurement [10] by Beams, but, on the other hand, it is $60-70$ times lower then the cavitation pressures deduced from the experiments with hemispherical piezoelectric transducers, where cavitation is assumed to be homogeneous. This fact allows us to conclude that in our case the observed cavitation is heterogeneous in nature.

\subsection{On the possible role of impurities, solid surfaces, radiation, ions and quantized vortices - heterogeneous nucleation}

It is known that in conventional liquids such as water dissolved gases lower the cavitation threshold significantly. However, in liquid helium all gases (except ${ }^{3} \mathrm{He}$ ) are frozen out below $4.2 \mathrm{~K}$ and might be present only in the form of small solid particles. If helium were in contact with a solid surface (e.g., surface of the quartz fork or of such a frozen gas particle), the energy barrier against nucleation via formation of a bubble would probably be smaller than that in the pure bulk. Taking into account that liquid helium wets almost any solid surface nearly ideally, significant lowering of the cavitation threshold on smooth surfaces is unlikely. However, a possibility of the existence of so-called «lobster pots» on rough surfaces containing various excrescences or cracks cannot be excluded and these could, in principle, lower the cavitation threshold significantly. Without detailed knowledge of the surface in question, any quantitative estimate is hardly possible.

Assuming that, in our case, cavitation occurs in the vicinity of the sharp corners on the fork's prongs, a simple estimate of the relevant liquid volume yields $V \approx \delta^{2} l \approx$ $\approx 10^{-15} \mathrm{~m}^{3}$, where $l$ denotes the characteristic size of the prong. This is even smaller then the estimated cavitation volume in the experiments with the hemispherical piezoelectric transducers thought to produce homogeneous cavitation. It is puzzling therefore why our cavitation threshold appears so low.

There is experimental evidence that negative ionsempty bubbles containing an electron-lower the cavitation threshold [16]. It is well known that a charged particle passing through a bubble chamber produces a track of bubbles. The underlying physics of this process in helium (and hydrogen) bubble chambers operating at low temperatures [25] seems to be rather mechanical then thermal. The authors of Ref. 25 used $\beta$ particles from a radio- 
active ${ }^{204} \mathrm{Tl}$ source that passed the miniature helium bubble chamber in which negative pressures were produced using a focused ultrasonic wave. An electron in liquid helium experiences a repulsive potential of about $1 \mathrm{eV}$ and it is thus energetically favorable to create a spherical cavity. The total energy $E$ of the bubble, i.e., of the electron and the cavity of radius $R$, in thin wall approximation, reads:

$$
E=E_{\mathrm{el}}+4 \pi R^{2} \alpha+\frac{4}{3} \pi R^{3} p+2 \pi R^{3} \rho \dot{R}^{2},
$$

where $\alpha$ denotes the surface tension, $p$ is the pressure and $\rho$ is the density of liquid helium. The terms on the RHS have the following meanings: $E_{\mathrm{el}}$ is the ground state energy of the electron in the cavity, $4 \pi R^{2} \alpha$ is the surface energy of the bubble, $(4 / 3) \pi R^{3} p$ represents the difference in the Gibbs free energy neglecting the vapor density and pressure (which is justified at low temperature) and the last term (which can be rewritten for clarity as $4 \pi R^{2} R \rho \dot{R}^{2} / 2$ ) represents the kinetic energy of the bubble when its size is changing at the rate $\dot{R}$ and the liquid is treated as incompressible.

As nicely explained by the authors of Ref. 25, the significance of the kinetic term in Eq. (1) is to carry the bubble radius past the state of minimum energy and thus overcome the potential barrier at a somewhat lower negative pressure of -0.69 bar. This estimate was found in good agreement with experimental value of -0.52 bar that hardly changed in the range $0.7 \mathrm{~K}<T<1.5 \mathrm{~K}$.

Note in passing that Eq. (1) involves the physics of (i) bubble «explosion» as well as (ii) homogeneous cavitation in thin wall approximation, simply by dropping the last (i) and additionally the first (ii) term on the RHS, yielding the corresponding negative pressure cavitation thresholds of about -2 bar for an electron and -9 bar for an empty bubble in the zero temperature limit.

The electron bubbles «explode» under an applied negative pressure of about -1 bar in He I below $4.2 \mathrm{~K}$, which gradually decreases with decreasing temperature to about -2 bar in He II. These negative pressures are still too high to be directly compared to our measurements and we are thus forced to consider other possibilities.

One of these is that cavitation can be associated with vorticity, as it was first proposed already in 1944 by Dean [26], and developed by McConnell, Chu and Finch [27], who considered a mechanism of ultrasonic cavitation in liquid helium by quantized vortices. It seems clear that the velocity field of a single quantized vortex is much too weak to affect the nucleation barrier appreciably. Although in our case cavitation occurs at velocities far beyond the critical velocity, in the turbulent drag regime, where a dense tangle of quantized vortices is present [24], a simple estimate shows that it still seems unlikely that many vorti- ces could exist within the volume determined by the critical cavitation bubble radius.

To conclude this discussion of our He II data, it seems clear that the observed cavitation is not homogeneous in nature. Further detailed investigations both theoretical and experimental would be necessary in order to determine which of the above mentioned possibilities is responsible for the low cavitation threshold observed in our experiments.

\subsection{Discussion of our He I data}

Contrary to the situation in He II discussed above, it is easy to show that overheating is significant in He I. Let us estimate the magnitude of this effect. In normal He I the measured critical cavitation velocity is about three to four times lower then in He II (below $1 \mathrm{~m} / \mathrm{s}$ ). The driving force (in the turbulent drag regime) is about twice lower [7], nevertheless, this strongly suggests (though without thermal convection taken into account) that in $\mathrm{He} \mathrm{I}$ it is most likely that combined boiling/cavitation rather than pure cavitation occurs. In other words, we actually measured cavitation that took place at a temperature significantly higher than $T_{0}$ at which the bath was kept.

This conclusion is consistent with the analysis of the data showing the measured cavitation velocity versus the applied overpressure (Fig. 9). The data sets are consistent with the Bernoulli equation, however, the logarithmic inset shows that such a fit is not quite conclusive. When forced, using the data for two forks, it gives values for the flow enhancement factors $k_{e 1}=3.1$ and $k_{e 2}=2.6$; i.e., lower than that obtained at $1.3 \mathrm{~K}$ in He II. The resulting cavitation pressure is very low, of order $100 \mathrm{~Pa}$ below the

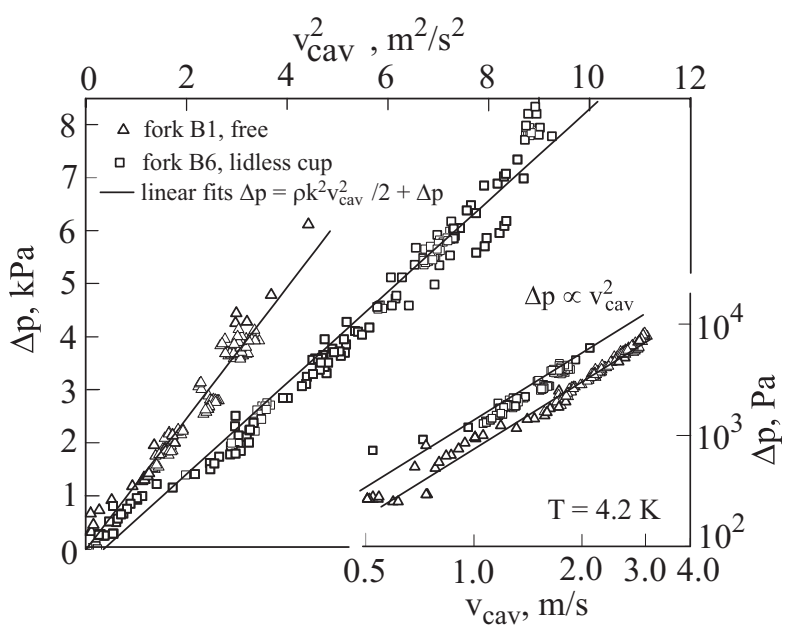

Fig. 9. The applied overpressure plotted versus the square of the observed critical cavitation velocity at $4.2 \mathrm{~K}$. The solid lines are the best fits to the Bernoulli equation yielding the flow enhancement factors $k_{e 1}=3.1$ and $k_{e 2}=2.6$ (compare to $k_{e}=4.7$ in He II). The inset shows the overpressure versus the cavitation velocity in logarithmic axes. The solid lines in the inset have a slope of 2 corresponding to the quadratic dependence in accord with the Bernoulli equation. 
saturated vapour pressure, i.e., comparable with the hydrostatic pressure head in the pressure cell.

\subsection{Discussion of cavitation data obtained in the vicinity of the superfluid transition}

We will now investigate the behavior of the critical cavitation velocity in the vicinity of the superfluid transition. A very pronounced feature - namely a steep increase of the critical cavitation velocity - can be seen in Fig. 3, just below $T_{\lambda}$. For the sake of a more precise quantitative discussion, the observed dependence was fitted by the Boltzmann sigmoidal function, of the form

$$
v(T)=v_{2}+\frac{v_{1}-v_{2}}{1+\exp \left[\left(T-T_{0}\right) / \Delta T\right]},
$$

and shown in detail in Fig. 10. The characteristic width of the increase was determined from the fit as $\Delta T \approx 20 \mathrm{mK}$.

As far as we know, there is no cavitation theory that would predict any pronounced sudden change of the cavitation threshold below the superfluid transition. However, as we discussed before, the main difference between the measurements in $\mathrm{He}$ I and He II is the degree of overheating of the vicinity of the vibrating fork. We have shown that it can probably be neglected in He II far below the $\lambda$-point but plays a significant part in cavitation observed with the fork in He I.

Let us consider the situation as it occurs upon cooling through the superfluid transition. Until the $\lambda$-point is reached from above, there is significant overheating and cavitation takes place at a higher temperature than that at which the bath is kept. The $\lambda$-anomaly in specific heat probably does not matter, as we are considering a steady-state situation; therefore we expect no qualitative

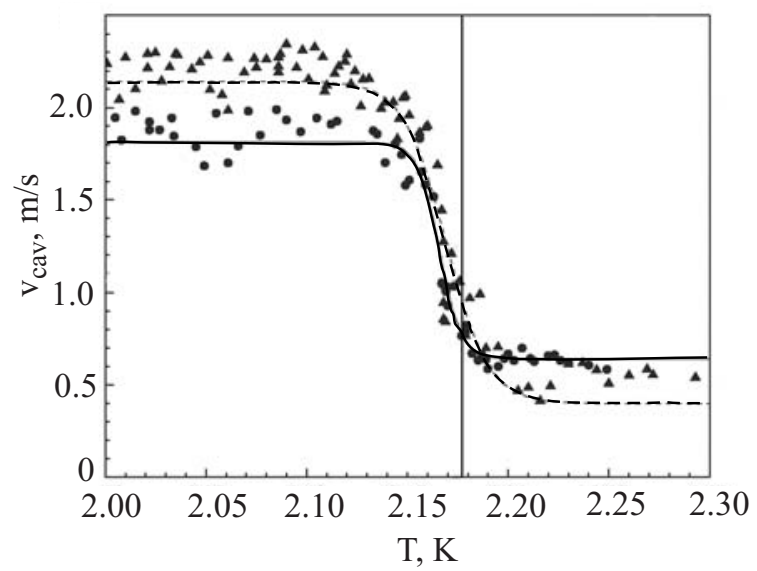

Fig. 10. The observed critical cavitation velocity at the saturated vapour pressure plotted versus the temperature in the vicinity of the superfluid transition temperature $T_{\lambda}$. The circles and the triangles represent two experimental runs (different days) obtained with the same partly encap-sulated fork inside the pressure cell. The solid and the dashed line are fits using the Boltzmann sigmoidal function (see text). change until $T_{\lambda}$. On crossing the superfluid transition (in the bath), in addition to the cooling channel due to the normal fluid (molecular conduction and thermal convection similar to that above the transition), a new cooling channel emerges - thermal counterflow. It is clear that this cooling channel becomes steeply more and more effective, although its efficiency is difficult to quantify. Important physical quantities such as superfluid to normal fluid density ratio, mutual friction coefficients, entropy and velocity of second sound are steep functions of temperature here [28]. Thus heat transfer is very nonlinear in nature at temperatures just below $T_{\lambda}$. For example, the mutual friction coefficient $\alpha$ is of order unity here, therefore in the situation with a dense tangle of vortex lines (in the turbulent drag regime) the normal and superfluid components are coupled strongly and the efficiency of counterflow heat transfer is suppressed. It seems therefore that the vicinity of the prongs could still be overheated significantly and the fork actually surrounded by $\mathrm{He} \mathrm{I}$, although the helium bath is kept at a temperature close below $T_{\lambda}$. Note in passing that the situation with the helium bath consisting of both He I and He II is indeed possible and is in fact commonly used as the so-called $\lambda$-point refrigerator.

Let us now discuss Fig. 6, showing the theoretical predictions for the temperature dependence of the cavitation threshold together with the available experimental data obtained with hemispherical piezoelectric transducers. These data are believed to represent homogeneous cavitation. We have added our own data, calculated assuming that the Bernoulli equation holds at any temperature and using the parameters of fits obtained at $1.3 \mathrm{~K}$. Such a procedure is certainly not quantitatively correct (we do not measure pure homogeneous cavitation), but we believe it illustrates the situation in the vicinity of $T_{\lambda}$ qualitatively. All experimental data appear qualitatively similar, displaying a pronounced kink around $T_{\lambda}$. This feature of the data looks very strange indeed, as theoretical calculations for cavitation predict no anomalies of this kind. A plausible explanation might again be that, in the experiments with hemispherical transducers, the cavitation spot is (similarly as in our fork experiments) overheated significantly. Indeed, acoustic pressurization - depressurization of extremely high amplitude cannot be strictly isothermal and it is plausible that significant overheating occurs in the acoustic center.

\section{Conclusions}

We have reported results on visual observation and electrical detection of cavitation in liquid ${ }^{4} \mathrm{He}$ that most likely takes place in the vicinity of a piezoelectric quartz tuning fork vibrating at a high amplitude. There is little doubt that the observed electrical effects are a signature of cavitation, although the understanding of the underly- 
ing physics requires to take thermal effects into account, especially in the vicinity of $T_{\lambda}$ and in normal liquid He I.

The analysis of our results based on the Bernoulli equation in He II, where thermal effects could most likely be neglected, strongly suggest that we deal with pure cavitation, albeit heterogeneous in nature. The deduced cavitation threshold is significantly lower than that observed in the experiments with hemispherical transducers, where cavitation occurs as a result of acoustic interference far away from walls and is generally believed to be homogeneous. We have discussed various mechanisms that could intervene in our case, leading to heterogeneous rather than homogeneous cavitation.

Based on the measured temperature dependence of the critical cavitation velocity that displays a pronounced steep increase on decreasing temperature within about $20 \mathrm{mK}$ below $T_{\lambda}$ we conclude that in He I the vicinity of the fork is locally overheated and cavitation occurs here at a significantly higher temperature than that at which the surrounding helium bath is kept. The steep increase of the cavitation velocity by a factor of 3-5 observed just below the superfluid transition can be understood as a consequence of the high convective heat transfer efficiency in superfluid He II compared to He I.

Our results and the presented discussion clearly show that the underlying physics of cavitation is only partly understood, even in a relatively simple - almost model system such as liquid ${ }^{4} \mathrm{He}$. We hope that this work will serve as a call for further experiments and theoretical investigations of the interesting cavitation phenomena in cryogenic helium liquids.

\section{Acknowledgements}

The authors appreciate the technical assistance of T.V. Chagovets, L. Doležal, M. Rotter, and P. Vacek; thank P. Bőhm for his professional help with cavitation photography and J. Pešička for providing the fork micrographs. This research is supported by the research plans MS 0021620834, AVOZ 10100520, by GAČR under 202/05/0218 and GAUK 7953/2007.

1. Q. Zheng, D.J. Durben, G.H. Wolf, and C.A. Angell, Science 254, 829 (1991).

2. H.J. Maris, S. Balibar, and M.S. Petersen, J. Low Temp. Phys. 93, 1069 (1993).

3. F. Caupin, S. Balibar, and H.J. Maris, Physica B329-333, $356(2003)$.
4. S. Balibar, J. Low Temp. Phys. 129, 363 (2002).

5. M. Blažková, M. Človečko, V.B. Eltsov, E. Gažo, R. de Graaf, J.J. Hosio, M. Krusius, D. Schmoranzer, W. Schoepe, L. Skrbek, P. Skyba, R.E. Solntsev, and W.F. Vinen, J. Low Temp. Phys. (in print).

6. R. Blaauwgeers, M. Blazkova, M. Clovecko, V.B. Eltsov, R. de Graaf, J. Hosio, M. Krusius, D. Schmoranzer, W. Schoepe, L. Skrbek, P. Skyba, R.E. Solntsev, and D.E. Zmeev, J. Low Temp. Phys. 146, 537 (2007).

7. M. Blazkova, D. Schmoranzer, and L. Skrbek, Phys. Rev. E75, 025302 (2007).

8. M. Blažková, M. Človečko, E. Gažo, L. Skrbek, and P. Skyba, J. Low Temp. Phys. 146, 305 (2007).

9. M. Blažková, T.V. Chagovets, M. Rotter, D. Schmoranzer, and L. Skrbek, J. Low Temp. Phys. (in print).

10. J.W. Beams, Phys. Rev. 104, 880 (1956).

11. R.D. Finch, R. Kagiwada, M. Barmatz, and I. Rudnick, Phys. Rev. 134, A1425 (1964).

12. P.D. Jarman, and K.J. Taylor, J. Low Temp. Phys. 2, 389 (1970).

13. P.L. Marston, J. Low Temp. Phys. 25, 383 (1976).

14. R.D. Finch and T.G.J. Wang, J. Acoust. Soc. Am. 39, 511 (1966).

15. R.D. Finch, T.G.J. Wang, R. Kagiwada, M. Barmatz, and I. Rudnick, J. Acoust. Soc. Am. 40, 211 (1966).

16. S.C. Hall, J. Classen, C.K. Su, and H.J. Maris, J. Low Temp. Phys. 101, 793 (1995).

17. M.S. Pettersen, S. Balibar, and H.J. Maris, Phys. Rev. B49, 12062 (1994).

18. J.A. Nissen, E. Bodegom, L.C. Brodie, and J.S. Semura, Phys. Rev. B40, 6617 (1989).

19. D.N. Sinha, J.S. Semura, and L.C. Brodie, Phys. Rev. A26, 1048 (1982).

20. F. Caupin and S. Balibar, Phys. Rev. B64, 064507 (2001).

21. M. Gilleumas, M. Pi, M. Barranco, J. Navarro, and M.A. Solis, Phys. Rev. B47, 9116 (1993).

22. M. Gilleumas, M. Barranco, D.M. Jezek, R.J. Lombard, and M. Pi, Phys. Rev. B54, 16135 (1996).

23. L.D. Landau and E.M. Lifshitz, Fluid Mechanics, Pergamon Press (1959).

24. M. Blažková, D. Schmoranzer, L. Skrbek, and W.F. Vinen, submitted to Phys. Rev. Lett.

25. D. Konstantinov, W. Homsi, J. Luzuriaga, C.-K. Su, M.A. Weilert, and H.J. Maris, J. Low Temp.Phys. 113, 485 (1998).

26. R.B. Dean, J. Appl. Phys. 15, 446 (1944).

27. P.M. McConnell, M.L. Chu, Jr., and R.D. Finch, Phys. Rev. A1, 411 (1970).

28. R.J. Donnelly and C.F. Barenghi, J. Phys. Chem. Data 27, 1217 (1998). 